\title{
New ionic liquids with hydrolytically stable anions as alternatives to hexafluorophosphate and tetrafluoroborate salts in the free radical polymerization and preparation of ion-conducting composites
}

\author{
Yakov S Vygodskii ${ }^{1}$, Dmitrii A Sapozhnikov ${ }^{1}$, Alexander S Shaplov ${ }^{1}$, Elena I Lozinskaya ${ }^{1}$, Nikolai V Ignat'ev ${ }^{2}$ \\ Michael Schulte ${ }^{2}$, Petr S Vlasov ${ }^{3}$ and Inna A Malyshkina ${ }^{4}$
}

The main objective of this study was to replace the traditionally used hydrolytically unstable $\mathrm{PF}_{6}$ and $\mathrm{BF}_{4}$ ionic solvents with new, stable ionic liquids (ILs) and to investigate the potential of ILs in formulation of the polymers based on methyl methacrylate and acrylonitrile with narrow polydispersities, high yields and molecular weights. Various ILs with four anions, namely, tris(pentafluoroethyl)trifluorophosphate, trifluoromethanesulfonate, trifluoroacetate and tetracyanoborate, were tested. The influence of the IL's characteristics on the free radical polymerization process was found to be significant. Trifluoromethanesulfonate- and trifluoroacetate-based ionic solvents provide the formation of polymers with the highest molecular weight $\left(M_{\mathrm{r}} \approx 8.8 \times 10^{5}\right.$ for polyacrylonitrile (PAN) and $M_{\mathrm{w}} \approx 18.9 \times 10^{5}$ for polymethylmethacrylate (PMMA)) in quantitative yield (PAN, 96\%; PMMA, 92\%). Taking into account, the high ionic conductivity and satisfactory electrochemical stability of the tetracyanoborate-based ILs, composite films derived from PAN and B(CN) ${ }_{4}$ ILs were prepared and their physico-chemical properties were investigated. The nature of the cation strongly influences the properties of the resulting composite films, namely, their flexibility, transparency, surface morphology and ionic conductivity (the maximum conductivity was found to be $1.0 \times 10^{-2} \mathrm{~S} \mathrm{~cm}^{-1}$ at $25^{\circ} \mathrm{C}$ ).

Polymer Journal (2011) 43, 126-135; doi:10.1038/pj.2010.115; published online 8 December 2010

Keywords: acrylonitrile; ionic conductivity; ionic liquids; methyl methacrylate; polymerization; polyelectrolyte

\section{INTRODUCTION}

In the recent years, the synthesis of polymers in ionic liquids (ILs) has attracted considerable attention. ${ }^{1-8}$ The increasing interest in ILs as reaction media ${ }^{9}$ and electrolytes ${ }^{10-12}$ is due to their unique properties (for example, nonvolatility, nonflammability, high ionic conductivity and good thermal and electrochemical stability) and to the peculiarities of the chemical reactions performed in ILs. ${ }^{1,9,13}$

In comparison with common organic solvents, ILs have high polarities and viscosities, ${ }^{9}$ and thus they accelerate the free radical polymerization of unsaturated monomers and support the formation of polymers with ultra-high molecular weights. ${ }^{3-6,14-17}$ The influence of ILs on the kinetics of polymerization processes is related to the decrease in the activation energy ${ }^{5,18,19}$ and the respective increase in the propagation rate. ${ }^{4,5,18-22}$ At the same time, the rate of termination decreases, $4,5,20,22,23$ sometimes even by an order of magnitude, with the increase of IL concentration. ${ }^{18}$ The increase in the propagation rate is generally attributed to the increased polarity of the ionic medium in comparison with that of the organic solvents that are traditionally used, whereas the decrease in the termination rate is assumed to be due to the increased viscosity of ILs. ${ }^{3-6,18,20,24,25}$ Andrzejewska et al. ${ }^{21}$ detected the phenomenon of viscosity synergism in dimethacrylate monomer/IL mixtures (that is, the viscosity of the mixture is higher than the simple additive combination of the viscosities of the two components); this phenomenon can have an important role in the kinetics of the reaction because the possibility of the gel effect is higher in the IL solution than in the bulk monomer. Schmidt-Naake et al..$^{5}$ partly explained the reduction of the activation energy of propagation by the specific interaction of ethyl acrylate with ILs and the formation of H-bonds between both the monomer molecules and growing radical chains and the cations and anions of the ILs. Irvine et al. ${ }^{6}$ proposed that a 'protected' radical mechanism occurs during polymerization in ILs; that is, some stabilization

${ }^{1}$ A.N. Nesmeyanov Institute of Organoelement Compounds Russian Academy of Sciences (INEOS RAS), Moscow, Russia; ${ }^{2}$ Merck KGaA, PC-RL, Darmstadt, Germany; ${ }^{3}$ Department of Macromolecular Chemistry, Saint-Petersburg State University, Universitetsky pr. 26, Saint-Petersburg, Russia and ${ }^{4}$ Department of Physics, Moscow State University, Vorob'evy gory, Moscow, Russia

Correspondence: Professor YS Vygodskii and Dr AS Shaplov, A.N. Nesmeyanov Institute of Organoelement Compounds Russian Academy of Sciences (INEOS RAS), Vavilov str. 28, GSP-1, Moscow 119991, Russia. 
of radical moieties in ionic solvents could take place. By investigating poly(ethylene glycol)dimethacrylates (PEGDMs) photopolymerization kinetics, Chesnokov and Shaplov ${ }^{26,27}$ found that the nature of ILs greatly influences both the reduced photopolymerization rate and the oligomer conversion. This influence is rather complicated and none of the commonly known properties of ILs, including the polarity, viscosity and volume of the anion, are able to solely describe or explain the established correlations. A satisfactory explanation can be given only by the acceptance of the assumption of presence of stable intermolecular structures with 'kinetically unfavorable' molecular arrangements in liquid monomers. The influence of IL addition on the $\operatorname{PEGDM}(n)$ photopolymerization kinetics consists of changing the supramolecular structures of both the monomer and the IL. ${ }^{26,27}$ The destruction of the monomer associates led mainly to the dramatic acceleration of the corresponding monomer polymerization. In contrast, the preservation of the supramolecular structure of the IL inside the monomer medium resulted either in the reinforcement of its 'kinetically unfavorable' association and, as a consequence, in the suppression of its polymerization or in the reinforcement of its 'kinetically favorable' association and a slight acceleration of the reaction. ${ }^{26,27}$

The major advantages of using of ILs in free radical polymerization are (a) their ability to dissolve a wide range of organic and ionic compounds, including monomers and polymers; (b) the acceleration of the polymerization reaction; (c) the safety of the process, in particular because of the nonflammability and nonvolatility of ILs and (d) the possibility of solvent recycling. These intriguing features have stimulated interest in the application of ILs as reaction media in other types of radical polymerization, including the reversible addition-fragmentation chain transfer ${ }^{28-31}$ process, polymerization with atom (atom transfer radical polymerization), ${ }^{32-44}$ group $^{45}$ or charge $e^{46}$ transfer, nitroxide-mediated polymerization ${ }^{47}$ and some others. ${ }^{48-50}$

The majority of published reports ${ }^{14,15,19,23,29,30,32,34,37,39,51}$ devoted to polymerization in ILs as reaction media only deals with the investigation of commonly known ILs with fluorinated anions such as tetrafluoroborate, hexafluorophosphate and bis(trifluoromethylsulfonyl)imide ILs. Indeed, such ILs have proven to be very promising reaction media for radical polymerization; they readily dissolve unsaturated monomers and provide access to high molecular weight polymers in good yields. However, $\mathrm{BF}_{4}$ and $\mathrm{PF}_{6}$ anions are not very stable hydrolytically. ${ }^{9,52}$ At the temperature of the polymerization processes $\left(>60^{\circ} \mathrm{C}\right)$, in the presence of traces of water, they can undergo hydrolysis and release unfavorable hydrofluoric acid (HF). In contrast, hydrolytically stable ILs with bis(trifluoromethylsulfonyl)imide anions are quite expensive, which reduces their practical application. For these reasons, in the presented work several new families of ILs with hydrolytically stable anions, such as tris(pentafluoroethyl)trifluorophosphate, ${ }^{53}$ trifluoroacetate, trifluoromethanesulfonate and tetracyanoborate, ${ }^{54}$ were investigated to replace the traditionally used $\mathrm{PF}_{6}$ and $\mathrm{BF}_{4}$ ionic solvents and to study their potential for polymer synthesis, in particular in the free radical polymerization of methyl methacrylate (MMA) and acrylonitrile (AN).

The nature of the cations in ILs may also influence the polymer formation in ionic media. 2,3,6,15,16,26,27,37,55 For example, the impact of the alkyl chain length and symmetry of the cation on the free radical polymerization of acrylic monomers in 1,3-dialkylimidazolium ILs has been studied and clearly demonstrated. ${ }^{6,14-16,37}$ Thus, in parallel with stable anions, different cations, namely, imidazolium, pyridinium, pyrrolidinium, ammonium, phosphonium, guanidinium and iso-uronium, were studied as well.

The application of ILs as dopants to the polymer matrix (see for example refs 56-59) or as a comonomer in the synthesis of polymeric materials ${ }^{60-63}$ creates new attractive opportunities for the development of ion-conducting materials, which are interesting for various applications. Among the suggested ILs, those with the tetracyanoborate anion possess the highest ionic conductivity. ${ }^{64}$ Therefore, in addition to the radical polymerization of $\mathrm{AN}$, the application of these ILs in the formation of ion-conducting films was also investigated.

\section{EXPERIMENTAL PROCEDURE}

\section{Materials}

MMA (Sigma-Aldrich Corp., St Louis, MO, USA, 99\%) and AN (SigmaAldrich Corp., $\geqslant 99 \%$ ) were distilled in a vacuum before use. 2,2'-Azobisisobutyronitrile (Acros Organics, Thermo Fisher Scientific, Geel, Belgium, 98\%) was purified via crystallization from methanol. ILs with purities of 98-99.5\% were obtained from Merck KGaA Company (Darmstadt, Germany). All ILs were thoroughly dried for $2-4 \mathrm{~h}$ at $50-55^{\circ} \mathrm{C}$ under a high vacuum $(<1$ Torr) with a special flask/trap filled with $\mathrm{P}_{2} \mathrm{O}_{5}$ and introduced into the vacuum line before their utilization.

\section{Polymerization of MMA and AN}

Freshly dried IL was charged with the solution of respective monomer and initiator-2, $2^{\prime}$-azobisisobutyronitrile ( $0.1 \mathrm{wt} \%$ for $\mathrm{AN}$; $0.5 \mathrm{wt} \%$ for MMA). The mass ratio between the monomer and the IL was 1:1. The ampoule with the reaction mixture was repeatedly frozen and evacuated and afterwards was sealed under a vacuum and placed into the thermostat. The polymerization was carried out for $6 \mathrm{~h}$ at $60^{\circ} \mathrm{C}$. The samples of polymethylmethacrylate (PMMA) were purified by precipitation from chloroform solution into methanol, whereas polyacrylonitrile (PAN) was purified by precipitation from DMF (dimethylformamide) into methanol. The polymers obtained were washed several times with methanol and dried in a vacuum $(<1$ Torr $)$ at $70{ }^{\circ} \mathrm{C}$ for $48 \mathrm{~h}$.

\section{Film formation}

Composite materials $(0.3 \mathrm{~g})$, produced by free radical polymerization as described above from AN and various tetracyanoborate ILs, were dissolved in $5 \mathrm{ml}$ of DMF. Films were cast from the prepared solutions on a glass support with further slow solvent evaporation at $70^{\circ} \mathrm{C}$. To remove the traces of AN and DMF, the composite films were thoroughly dried in a vacuum $(<1$ Torr $)$ at $70^{\circ} \mathrm{C}$ for $48 \mathrm{~h}$.

The second method of the film formation included the preparation of PAN in one of the ILs as described above and its consequent isolation by precipitation into methanol, filtration and complete drying in a vacuum $(<1$ Torr) at $70{ }^{\circ} \mathrm{C}$ for $48 \mathrm{~h}$. The pure PAN $(0.2 \mathrm{~g})$ obtained was re-dissolved in $3 \mathrm{ml}$ of DMF and the desired amount of selected IL was added $0.2 \mathrm{~g}$ for $50 \mathrm{wt} \%$ containing conductive film) to the solution. After thorough stirring, the transparent solution was poured onto the glass support and the solvent was slowly evaporated at $70^{\circ} \mathrm{C}$. The obtained composite films were dried in a vacuum $\left(<1\right.$ Torr) at $70^{\circ} \mathrm{C}$ for $48 \mathrm{~h}$.

\section{Measurements}

The inherent viscosities $\left(\eta_{\text {inh }}\right)$ of the polymers were measured at $25.0^{\circ} \mathrm{C}$ using solutions of $0.05 \mathrm{~g}$ of the polymer in $10 \mathrm{ml}$ solvent (chloroform for PMMA and DMF for PAN samples) in an Ostwald capillary viscosimeter. The relative polymer molecular mass $\left(M_{\mathrm{r}}\right)$ of PAN was calculated according to formula 1:

$$
[\eta]=K \cdot\left(M_{\mathrm{r}}\right)^{\alpha}
$$

where $[\eta]$ is the polymer intrinsic viscosity. For the solution of PAN in DMF, $K=2.33 \times 10^{-4}$ and $\alpha=0.75{ }^{65}$ The relative molecular mass (molecular weight) is recommended for this equation rather than the molar mass (which has the dimension of mass divided by the amount of substance) because, in the latter case, the constant $K$ assumes awkward and variable dimensions due to the fractional and variable nature of the exponent $\alpha .{ }^{65}$

The molecular weights $\left(M_{\mathrm{n}}, M_{\mathrm{w}}\right)$ and polydispersity indexes $\left(M_{\mathrm{w}} / M_{\mathrm{n}}\right)$ of the PMMA samples were determined at $40^{\circ} \mathrm{C}$ on a LC-20AD gel permeation chromatograph (Shimadzu Corporation, Kyoto, Japan) equipped with a Tosoh TSK-GEL G5000Hhr column and a refractive index detector. Tetrahydrofuran was used as an eluent with a flow rate of $0.6 \mathrm{ml} \mathrm{min}^{-1}$. The calibration was performed on the basis of polystyrene standards (Fluka, Sigma-Aldrich Chemie GmbH, Munich, Germany). 
The mechanical properties of the films, that is, $\delta$ (tensile strength), $\varepsilon$ (elongation) and $E$ (elastic modulus), were measured at $25^{\circ} \mathrm{C}$ using a Polyani dynamometer (Polyani, Hungary).

The atomic force microscopy images were obtained using Scanning Tunneling Microscope Solver-P47 (ZAO 'NT-MDT', Zelenograd, Russia) in the semi-contact topographical mode The NSG11 cantilever (ZAO 'NT-MDT', Zelenograd, Russia) with a $2.5-10 \mathrm{~N} \mathrm{~m}^{-1}$ hardness (typically $5.5 \mathrm{~N} \mathrm{~m}^{-1}$ ) and with a resonance frequency equal to $\sim 193 \mathrm{kHz}$ was used. The samples for atomic force microscopy investigations were prepared as films by the method described above.

Dielectric spectroscopy studies were run on a Novocontrol Broadband Dielectric Spectrometer equipped with an Alpha analyzer and a Quatro temperature controller (Novocontrol Technologies GmbH \& Co. KG, Hundsangen, Germany). Polymer films were placed between the two gold-plated brass electrodes. The experiments were carried out isothermally at $25^{\circ} \mathrm{C}$ by varying the frequency from $10^{-1}$ to $10^{7} \mathrm{~Hz}$.

\section{RESULTS AND DISCUSSION}

\section{Free radical polymerization in ILs}

Generally, ILs applications as reaction media in both organic and polymer synthesis have been intensively studied with the use of commonly known ionic solvents containing $\mathrm{BF}_{4}$ and $\mathrm{PF}_{6}{ }^{1,2,9}$ likely because of the wide liquid range and good dilution ability of these ILs. However, Rogers et al. ${ }^{52}$ established that hexafluorophosphate imidazolium ILs are especially subjected to hydrolysis even in the presence of water traces. The HF eliminated, in its turn, can participate in the acidic hydrolysis of some monomers, thus limiting the utilization of ILs. The first two families of ionic solvents, namely, tetracyanoborate and tris(pentafluoroethyl)trifluorophosphate (FAP) ILs, have been suggested as structural analogs to the salts containing $\mathrm{BF}_{4}$ and $\mathrm{PF}_{6}$ (Scheme 1). Trifluoroacetate and trifluoromethanesulfonate ILs were chosen because of their lower cost and simplicity of preparation in comparison with bis(trifluoromethylsulfonyl)imide ILs.

As mentioned above, the nature of the cation also has an important role in the polymerization processes in ILs. Thus, along with the newly proposed anions, a wide range of cations was chosen (Table 1). However, it was not possible to synthesize all the ion combinations and thus, some ILs were not tested.

MMA and AN solubility tests were carried out before the polymerization runs. Almost all proposed ILs (Table 1) were capable of dissolving of MMA and $\mathrm{AN}$ in a 1:1 ratio, by weight, with the exception of some solvents containing $\mathrm{B}(\mathrm{CN})_{4}$. The appearance of small traces of water in tetracyanoborate ILs during their storage could prevent the dissolution of MMA in a 1:1 ratio by weight. However, when the ILs with $\mathrm{B}(\mathrm{CN})_{4}$ (Table 1, entries 4 and 10) were additionally dried, it became possible to dissolve MMA in the mentioned proportion.

MMA and AN polymerization was performed under optimal conditions, ${ }^{56,67}$ namely, the experiments were run at $60^{\circ} \mathrm{C}$ for $6 \mathrm{~h}$ with an $2,2^{\prime}$-azobisisobutyronitrile concentration equal to $0.1 \mathrm{wt} \%$ for $\mathrm{AN}$ and $0.5 \mathrm{wt} \%$ for MMA. Depending on the nature of the IL used, the obtained PMMA blocks, containing $50 \mathrm{wt} \%$ of ionic solvent, were either transparent (colorless or tinged) or cloudy (white or beige). The precipitation of PMMA occurred upon polymerization in all ILs containing trifluoroacetate and in trioctylmethylammonium trifluoromethanesulfonate salt with the formation of white or beige opaque samples. However, the purification of PMMA by re-precipitation always resulted in the formation of white powder. In contrast to PMMA, the precipitation of PAN during polymerization was observed in all ILs tested. Interestingly, the isolated PAN, irrespective of its color (from white to light brown) or of the IL used for its synthesis, was completely soluble in DMF. In comparison to polymerization in
ILs, the bulk polymerization of AN, initiated by $2,2^{\prime}$-azobisisobutyronitrile, ordinarily leads to the formation of a crosslinked polymer that is insoluble in DMF.

As a simple measurement to compare the molecular weights of the PMMA samples, the inherent viscosities of the polymer solutions in chloroform at $25.0{ }^{\circ} \mathrm{C}$ were determined. ${ }^{65}$ As shown in Table 1 , entries $1-3,6-9$ and 11 , the highest PMMA molecular weights were generally achieved in ILs bearing the trifluoromethanesulfonate anion, which is in a good agreement with the data obtained previously for other salts containing $\mathrm{CF}_{3} \mathrm{SO}_{3} .{ }^{14}$ The influence of the anions on the molecular weight of PMMA is clearly demonstrated with comparison of ionic solvents with similar cations (Table 1, entries 1, 3, 6, 7 and 11). Thus, the anions investigated can be placed in the following order: $\mathrm{CF}_{3} \mathrm{SO}_{3}>\mathrm{CF}_{3} \mathrm{COO}>\mathrm{PF}_{3}\left(\mathrm{C}_{2} \mathrm{~F}_{5}\right)_{3} \gg \mathrm{B}(\mathrm{CN})_{4}$, where tetracyanoborate anion allows for the synthesis of PMMA with the lowest molecular weight. The impact of the length of the side alkyl chain in imidazolium-based trifluoromethanesulfonate ILs was significant (Table 1, entries 1-3). The increase in the number of carbon atoms from $C_{2}$ to $\mathrm{C}_{4}$ caused the viscosity of PMMA to decrease from 510 to $440 \mathrm{mlg}^{-1}$ (Table 1, entries 1 and 2). The substitution of the methyl group for the ethyl group in the 1-butyl-3-alkyl imidazolium trifluoromethanesulfonate IL led to a further decrease in PMMA viscosity up to $380 \mathrm{ml} \mathrm{g}^{-1}$ (Table 1, entries 2 and 3). In contrast to ILs bearing $\mathrm{CF}_{3} \mathrm{SO}_{3}$, the change of the alkyl side chain length in FAP-based ionic solvents did not significantly affect the molecular weight of PMMA (Table 1, entries 1, 2 and 5). The ILs containing trifluoroacetate did not follow the established dependence, which can be explained by the precipitation of PMMA during polymerization. Noticeably, the cations of the trifluoromethanesulfonate ILs can be arranged as follows (Scheme 2) according to their influence on the molecular weight of PMMA.

This order partly coincides with the data published by Strehmel et al. ${ }^{15}$ where it was shown that the free radical polymerization of $n$-butyl methacrylate proceeded with higher $M_{\mathrm{w}}$ in imidazolium ILs compared with pyridinium ones. The order obtained was not relevant for tris(pentafluoroethyl)trifluorophosphate ILs, where the difference between the measured PMMA inherent viscosities was quite small $\left(20-40 \mathrm{ml} \mathrm{g}^{-1}\right)$.

The PMMA yields varied markedly (from 76 to $98 \%$ ) with the specific ionic solvent used for polymer synthesis, although the conditions were always the same. However, the examination of the impact of ILs was rather polysemantic (Table 1). Thus, for trifluoromethanesulfonate ILs, the increase in the length of the side alkyl chain in imidazolium solvents led to an increase in the PMMA yield (Table 1, entries 1-3). At the same time, the order of $\mathrm{CF}_{3} \mathrm{SO}_{3}$-bearing ILs according to their influence on PMMA yield did not agree with the<smiles>N#C[Sb](C#N)(C#N)C#N</smiles><smiles>FP(F)(F)(F)(F)(F)F</smiles><smiles>FC(F)(F)C(F)(F)C(F)(F)C(F)(F)P(F)(F)(C(F)(F)F)C(F)(F)C(F)(F)F</smiles>

Scheme 1 
Table 1 Influence of IL's nature on the yield and inherent viscosity of the polymers obtained $\left(60{ }^{\circ} \mathrm{C}, 6 \mathrm{~h},(\mathrm{AIBN})=0.1 \mathrm{wt} \%\right.$ for $\mathrm{AN}$ and $0.5 \mathrm{wt} \%$ for MMA; monomer/LL=1:1, by weight)

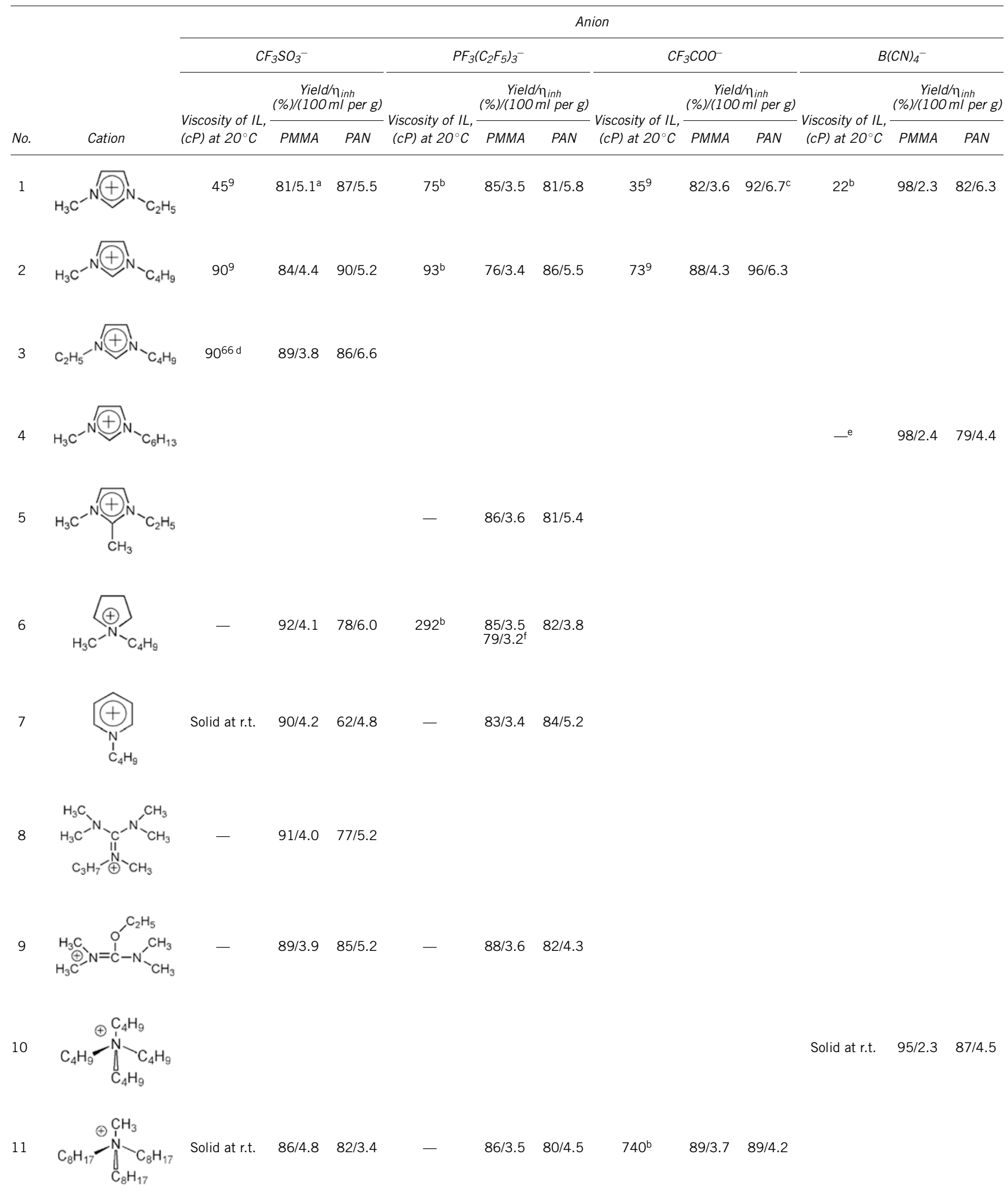




\begin{tabular}{|c|c|c|c|c|c|c|c|c|c|c|c|c|c|}
\hline \multirow[b]{3}{*}{ No. } & \multirow[b]{3}{*}{ Cation } & \multicolumn{12}{|c|}{ Anion } \\
\hline & & \multirow[b]{2}{*}{$\begin{array}{l}\text { Viscosity of IL, } \\
(c P) \text { at } 20^{\circ} \mathrm{C}\end{array}$} & \multicolumn{2}{|c|}{$\begin{array}{l}Y i e l d / \eta_{\text {inh }} \\
(\%) /(100 \mathrm{ml} \text { per } \mathrm{g})\end{array}$} & \multirow[b]{2}{*}{$\begin{array}{l}\text { Viscosity of IL, } \\
\text { (cP) at } 20^{\circ} \mathrm{C}\end{array}$} & \multicolumn{2}{|c|}{$\begin{array}{l}\text { Yield// } \text { inh }_{\text {inh }} \text { (\%) } \\
(\%(100 \mathrm{ml} \text { per })\end{array}$} & \multicolumn{3}{|c|}{$\begin{array}{c}Y i e l d / m_{\text {inh }} \\
(\%) /(100 \mathrm{ml} \text { per } \mathrm{g})\end{array}$} & \multicolumn{3}{|c|}{$\begin{array}{c}Y i e l d / \eta_{\text {inh }} \\
(\%) /(100 \mathrm{ml} \text { per } \mathrm{g})\end{array}$} \\
\hline & & & $P M M A$ & $P A N$ & & PMMA & PAN & $\begin{array}{l}\text {-Viscosity of IL, } \\
\text { (cP) at } 20^{\circ} \mathrm{C}\end{array}$ & $P M M A$ & PAN & $\begin{array}{l}\text { Viscosity of IL, } \\
(\mathrm{cP}) \text { at } 20^{\circ} \mathrm{C}\end{array}$ & $P M M A$ & PAN \\
\hline
\end{tabular}

Abbreviations: AIBN, azobisisobutyronitrile; AN, acrylonitrile; DMF, dimethylformamide; IL, ionic liquid; MMA, methyl methacrylate; PMMA, polymethylmethacrylate; PAN, polyacrylonitrile; r.t., room temperature.

aFor the solution of $0.05 \mathrm{~g}$ of PMMA in $10.0 \mathrm{ml}$ of chloroform or $0.05 \mathrm{~g}$ of PAN in $10.0 \mathrm{ml}$ of DMF at $25.0^{\circ} \mathrm{C}$.

bhttp://www.merck-chemicals.com/ionic-liquids/c_glib.s1Lhu4AAAEWqOEfVhTI.

cCalculated relative molecular mass of PAN was found to be $M_{\mathrm{r}} \approx 8.8 \times 10^{5}$ (based on the measured PAN's intristic viscosity [n]).

${ }^{\mathrm{d}}$ At $25^{\circ} \mathrm{C}$.

e.-' means could not be found in the literature.

fPolymerization was performed on air at atmospheric pressure.

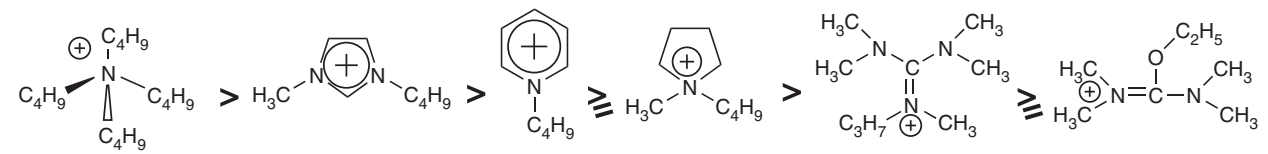

Scheme 2

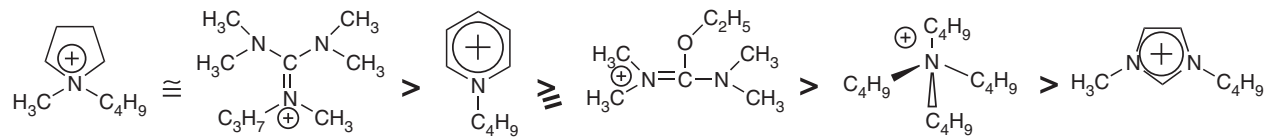

Scheme 3

order established by considering their influence on the PMMA molecular weight (Scheme 3).

The same sequence was found to be true for trifluoroacetatecontaining ILs (Table 1, entries 1, 2 and 11). With the exception of 1-butyl-3-methyl imidazolium tris(pentafluoroethyl)trifluorophosphate, the FAP-based ionic solvents always allowed for the synthesis of PMMA in $85-88 \%$ yield independently of the cation used. Moreover, the enhanced stability of the FAP ionic solvents promoted the realization of MMA polymerization even in air at atmospheric pressure without a practical decrease in the polymer molecular weight (Table 1, entries 6 and 12). The results (Table 1) show that, despite the average values of the molecular weights of PMMA obtained in tetracyanoborate ionic solvents, the PMMA yields in the mentioned ILs were always higher than in the others and varied in the range 95-98\% (Table 1, entries 1, 4 and 10) independently of the cations used.

For a more detailed investigation, gel permeation chromatography experiments were performed, and the molecular weight of PMMA was estimated for some experiments (Table 2). It should be emphasized here that the gel permeation chromatography measurements of PMMA were conducted in tetrahydrofuran and the molecular weights were determined from the calibration versus poly(styrene) standards. Therefore, values for $M_{\mathrm{n}}$ and $M_{\mathrm{w}}$ obtained for PMMA must be discussed with some reservation. The 1-butyl-3-methyl imidazolium cation was chosen as an example. The influence of the ILs was studied in comparison with the traditionally used salts containing $\mathrm{PF}_{6}$ and $\mathrm{BF}_{4}$ anions. The MMA polymerization performed in 1-butyl-3-methylimidazolium hexafluorophosphate and tetrafluoroborate resulted in
Table 2 GPC results for the synthesized PMMA

\begin{tabular}{|c|c|c|c|c|c|}
\hline \multirow[b]{2}{*}{ No. } & \multicolumn{2}{|c|}{ Ionic liquid } & \multicolumn{3}{|c|}{ PMMA molecular weights } \\
\hline & Cation & Anion & $\mathrm{M}_{w} \times 10^{-5}$ & $\mathrm{M}_{n} \times 10^{-5}$ & $\mathrm{M}_{w} \mathrm{M}_{n}^{\mathrm{a}}$ \\
\hline 1 & & $\mathrm{CF}_{3} \mathrm{SO}_{3}$ & 15.1 & 7.5 & 2.1 \\
\hline 2 & & $\mathrm{CF}_{3} \mathrm{COO}$ & 13.2 & 3.8 & $3.5^{\mathrm{b}}$ \\
\hline 3 & & $\mathrm{PF}_{3}\left(\mathrm{C}_{2} \mathrm{~F}_{5}\right)_{3}$ & 12.3 & 5.2 & 2.3 \\
\hline 4 & & $\mathrm{PF}_{6}$ & 12.2 & 6.3 & 2.0 \\
\hline 5 & & $\mathrm{BF}_{4}$ & 9.5 & 2.6 & $3.7^{b}$ \\
\hline 6 & & $\mathrm{CF}_{3} \mathrm{SO}_{3}$ & 17.8 & 10.9 & 1.6 \\
\hline 7 & & $\mathrm{~B}(\mathrm{CN})_{4}$ & 9.2 & 3.9 & 2.3 \\
\hline 8 & & $\mathrm{CF}_{3} \mathrm{SO}_{3}$ & 18.9 & 11.3 & $1.7^{b}$ \\
\hline
\end{tabular}

Abbreviations: GPC, gel permeation chromatography; PMMA, polymethylmethacrylate. aMeasured with polystyrene standards.

DPMMA precipitated during the polymerization.

polymer formation in 87 and $86 \%$ yield with $\eta_{\text {inh }}=290$ and $270 \mathrm{mlg}^{-1}$, respectively. Noticeably, [1-Me-3-Buim] $\mathrm{BF}_{4}$ was the only ionic solvent in which MMA was poorly soluble and the polymerization was carried out with a $\mathrm{MMA} / \mathrm{IL}=1: 3$ ratio. As shown in Table 2, 
entries 1-5, the polymer $M_{\mathrm{w}}$ obtained in all ILs bearing $\mathrm{CF}_{3} \mathrm{SO}_{3}$, $\mathrm{CF}_{3} \mathrm{COO}$ or $\mathrm{PF}_{3}\left(\mathrm{C}_{2} \mathrm{~F}_{5}\right)_{3}$ anions were higher than those in traditional salts containing $\mathrm{PF}_{6}$ and $\mathrm{BF}_{4}$. Interestingly, [1-Me-3Etim $] \mathrm{B}(\mathrm{CN})_{4}$ allowed for the synthesis of PMMA with a $M_{\mathrm{w}}$ nearly equal to that obtained in [1-Me-3-Buim] $\mathrm{BF}_{4}$ with a similar structure of the anion (Table 2, entries 5 and 7). In contrast to reference Strehmel et al., ${ }^{6}$ whether the polymerization was in solution or in precipitation was observed, the molecular weight distributions were found to be unimodal. However, on the application of trifluoroacetate and tetrafluoroborate ILs, the polydispersity indexes were rather high due to the PMMA precipitation (Table 2, entries 2 and 5). In terms of the molecular weights of the polymers and their distributions, FAPcontaining ILs could serve as a good alternative for their structural analogs, namely, for $\mathrm{PF}_{6}$-bearing ionic solvents (Table 2, entries 3 and 4). Finally, among the ILs studied, the 1-ethyl-3-methyl imidazolium and trioctylmethylammonium trifluoromethanesulfonates allowed for the synthesis of PMMA with the highest molecular weights, equal to $M_{\mathrm{w}}=17.8 \times 10^{5}$ and $18.9 \times 10^{5} \mathrm{~g} \mathrm{~mol}^{-1}$, respectively and with rather narrow polydispersity indexes (Table 2, entries 6 and 8 ).

In contrast to PMMA, PAN produced in trifluoroacetate ILs had the highest molecular weight (Table 1 , entries 1 and 2 ). The relative molecular mass calculated by the Mark-Kuhn-Houwink equation for the PAN synthesized in 1-ethyl-3-methylimidazolium trifluoroacetate was as high as $M_{\mathrm{r}}=8.8 \times 10^{5}$. In terms of the influence of the anion, the ILs can be arranged in the following order: $\mathrm{CF}_{3} \mathrm{COO}>\mathrm{B}(\mathrm{CN})_{4}>$ $\mathrm{PF}_{3}\left(\mathrm{C}_{2} \mathrm{~F}_{5}\right)_{3}>\mathrm{CF}_{3} \mathrm{SO}_{3}$, which does not coincide with what was found for PMMA. For comparison, AN was polymerized in [1-Me3-Buim] $\mathrm{BF}_{4}$ and [1-Me-3-Buim] $\mathrm{PF}_{6}$ using the same conditions as above. The results achieved in 'traditional' ILs, namely, PAN $\eta_{\text {inh }}=250 \mathrm{ml} \mathrm{g}^{-1}$ with $29 \%$ polymer yield and $\eta_{\text {inh }}=390 \mathrm{ml} \mathrm{g}^{-1}$ with $64 \%$ polymer yield in hexafluorophosphate and tetrafluoroborate salts, respectively, were worse than the results in the proposed ionic solvents (Table 1, entries 1 and 2).

Based on several identical experiments, it was possible to conclude that the increase in the length of the alkyl side chain leads to the decrease of the PAN viscosity irrespective of the IL's anion (Table 1, entries 1-3). Further investigation of the influence of the cation on the molecular weight of PAN resulted in the creation of two orders, one for ILs containing $\mathrm{CF}_{3} \mathrm{SO}_{3}$ (Scheme 4) and another for ILs bearing FAP (Scheme 5). The PAN yield showed no clear dependence on the structure of the IL used. The polymer yields were typically $80-85 \%$ (Table 1).

Finally, when comparing MMA and AN polymerization, no exact correlation between the polymer molecular weight and the viscosity of the starting ionic solvent was found, in contrast to previously published data. ${ }^{3,5,21}$ For comparison, the bulk viscosities of some of the ILs investigated are presented in Table 1. All the proposed ionic solvents that appear to be solid at $25^{\circ} \mathrm{C}$ are marked. The IL's viscosity data shown in Table 1 were found in the literature and the values are given where possible. Although some information is missing, the results can be compared. For example, the viscosities of 1-ethyl-3methyl imidazolium tris(pentafluoroethyl)trifluorophosphate and trihexyltetradecylphosphonium tris(pentafluoroethyl)trifluorophosphate are 75 and $464 \mathrm{cP}$ at $20^{\circ} \mathrm{C}$, respectively, whereas PMMA was obtained in both, with practically the same molecular weight (Table 1 , entries 1 and 12). Moreover, PAN synthesized in $\left[\mathrm{P}\left(\mathrm{C}_{6} \mathrm{H}_{13}\right)_{3}\left(\mathrm{C}_{14} \mathrm{H}_{29}\right)\right]$ $\mathrm{PF}_{3}\left(\mathrm{C}_{2} \mathrm{~F}_{5}\right)_{3}$ possessed even lower inherent viscosity than that obtained in [1-Me-3-Etim] $\mathrm{PF}_{3}\left(\mathrm{C}_{2} \mathrm{~F}_{5}\right)_{3}$.

Whether a particular monomer in a given solvent can be converted into a polymer depends on both thermodynamic and kinetic considerations. ${ }^{68}$ As it is well known, ${ }^{68}$ the free radical polymerization process is dependent on many factors, including (1) the solubility of the monomer and initiator in the given solvent, (2) the solubility of the resultant polymer in the mixture of monomer/solvent, (3) the rate of the main reactions (initiation, propagation) and (4) the rate of the termination reactions (combination (coupling) or disproportionation of radicals and the chain transfer). Thus, free radical polymerization, in particular with the use of ILs as reaction solvents, can be regarded as a complex multiple-factor process. To explain in detail the influence of ionic solvents on the polymer molecular weight and reaction rate, many additional experiments must be performed, including separate studies of the kinetics, solubility parameters and theoretical calculations. In contrast, the aim of this work was to compare the new families of ILs with those traditionally studied in terms of their utilization as solvents in MMA and AN radical polymerization. The total effect of the ILs is summarized in terms of the collation of yield and molecular weight of the polymers produced. Thus, based on these experiments, it is possible to conclude that, among the ILs tested, the imidazolium-based ILs generally produce polymers with higher molecular weights. Similar to reference Chesnokov et al., ${ }^{27}$ no 'common' rule was found for the choice of the best IL for free radical polymerization and, on the contrary, each monomer requires the selection of a definite ionic solvent.

\section{Composite films}

Among the newly proposed ILs, it is necessary to indicate those bearing the tetracyanoborate anion $\left[\mathrm{B}(\mathrm{CN})_{4}\right]$, which is a structural analog of the well-known tetrafluoroborate anion. The replacement of all four fluorine atoms in the $\left[\mathrm{BF}_{4}\right]$ anion with cyano groups leads to significant changes in the properties of the respective ILs. ${ }^{64}$ Ionic solvents with the tetracyanoborate anion are poorly soluble in water, less viscous and are more thermally and electrochemically stable than ILs with the tetrafluoroborate anion. ${ }^{64}$ However, tetracyanoborate ILs possess ionic conductivity similar to that of their tetrafluoroborate

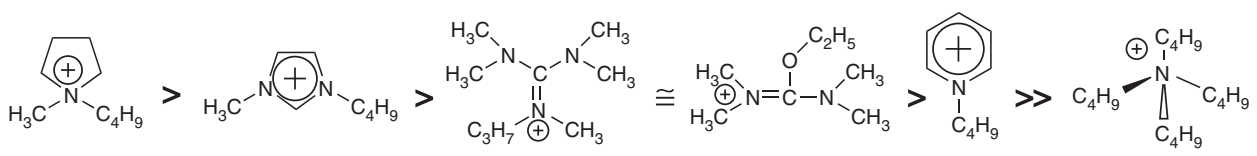

Scheme 4

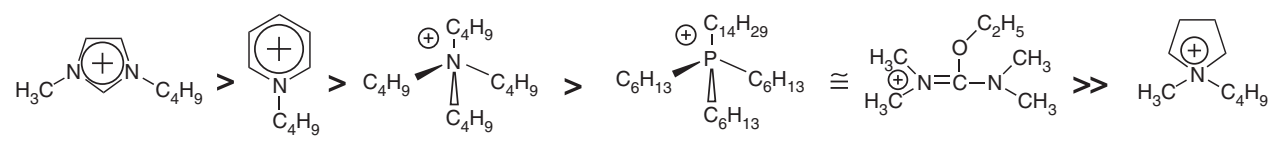


Table 3 The properties of PAN prepared in tetracyanoborates ionic liquids and the composite films therefrom

Mechanical properties of composite films

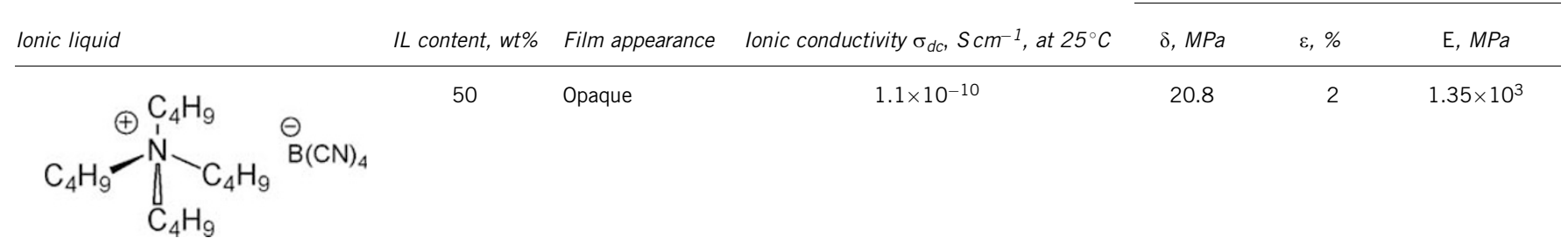

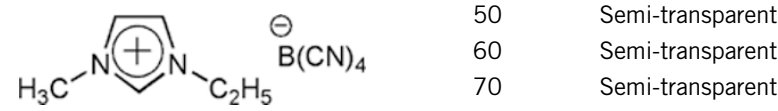

50

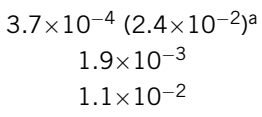<smiles></smiles>

$$
-\mathrm{b}
$$

- $\quad$ Transparent

Abbreviations: $\delta$, tensile strength; $\varepsilon$, elongation; $E$, tensile modulus; IL, ionic liquid; PAN, polyacrylonitrile.

alonic coductivity of the corresponding ionic liquid at $25^{\circ} \mathrm{C}$; for comparison reasons.

bPure PAN without filler is cited for comparison.

analogs (at $25^{\circ} \mathrm{C}, \sigma$ for $[1-\mathrm{Me}-3$-Etim $] \mathrm{BF}_{4}=1.7 \times 10^{-2} \mathrm{~S} \mathrm{~cm}^{-7}$, whereas $\sigma$ for $[1-\mathrm{Me}-3$-Etim $\left.] \mathrm{B}(\mathrm{CN})_{4}=2.4 \times 10^{-2} \mathrm{~S} \mathrm{~cm}^{-1}\right)$. Given that ILs containing $\mathrm{B}(\mathrm{CN})_{4}$ readily dissolved $\mathrm{AN}$ and formed semi-transparent composite films in combination with PAN, both the mechanical properties and the ionic conductivity of the film materials were investigated (Table 3).

Three tetracyanoborate ILs with ammonium and imidazolium cations were selected for study. $\left[\mathrm{N}\left(\mathrm{C}_{4} \mathrm{H}_{9}\right)_{4}\right] \mathrm{B}(\mathrm{CN})_{4}$ was chosen because the ammonium ILs are more electrochemically stable toward various metals, especially to lithium metal electrodes. ${ }^{69}$ However, imidazolium salts seem to have much lower viscosities and melting points as well as higher ionic conductivities (http://www.merckchemicals.com/ionic-liquids/c_glib.s1Lhu4AAAEWqOEfVhTl). The film cast from PAN and tetrabutylammonium IL was found to be fragile and opaque (Figure 1b), in contrast, films made from PAN and imidazolium tetracyanoborates were flexible, elastic and semi-transparent (Figures $1 \mathrm{c}$ and d). This difference could be explained either by the poor compatibility of the components in the case of tetrabutylammonium tetracyanoborate or by the possible complexing between the polymer chain and IL molecules, as in stereospecific PMMA formation. ${ }^{70}$ For further understanding, atomic force microscopy was utilized to study the surface of the composite films (Figure 2). The morphology of the films filled with imidazolium ILs (Figures 2c and d) was significantly different from that of the film containing tetrabutylammonium tetracyanoborate (Figure $2 \mathrm{~b}$ ). The latter had a 'needle' structure that was likely due to the poor compatibility of the polymer with the IL. In the case of imidazolium ILs (Figures $2 \mathrm{c}$ and d), PAN had a peculiar semi-swelled state, which could explain the elasticity and semi-transparency of the films obtained. The surface structure of such composite films (Figures $2 \mathrm{c}$ and d) is likely to be similar to the morphology of the films cast from pure PAN (Figure 2a).
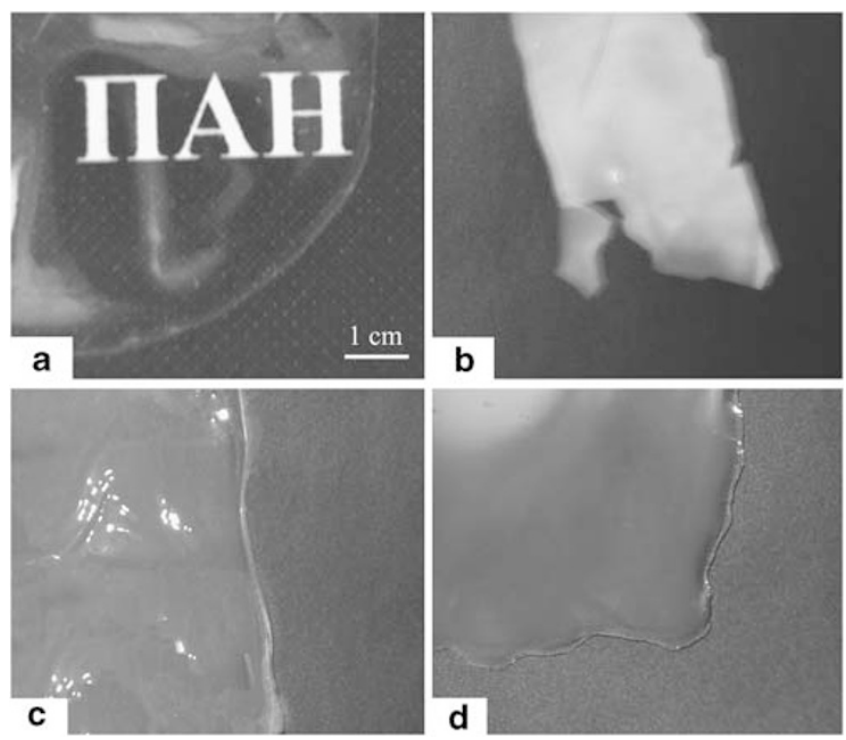

Figure 1 The appearance of the thin polymer films cast from standard polyacrylonitrile (PAN) without any filler (a)* and from PAN containing 50 wt $\%$ tetrabutylammonium tetracyanoborate (b), 1-ethyl-3-methylimidazolium tetracyanoborate (c) or 1-hexyl-3-methylimidazolium tetracyanoborate (d); ${ }^{*} \Pi \mathrm{AH}$ means PAN in Russian.

The ionic conductivity of composite films consisting of PAN and tetracyanoborate ILs was studied at $25^{\circ} \mathrm{C}$ in a broad range of frequencies from 50 to $10^{7} \mathrm{~Hz}$ (Figures 3 and 4 and Table 3 ). In general, the conductivity spectra of disordered systems show a plateau in $\sigma^{\prime}$ (giving the $\sigma_{\mathrm{dc}}$ value, that is the conductivity in the dc limit, or the direct current conductivity), which is followed 


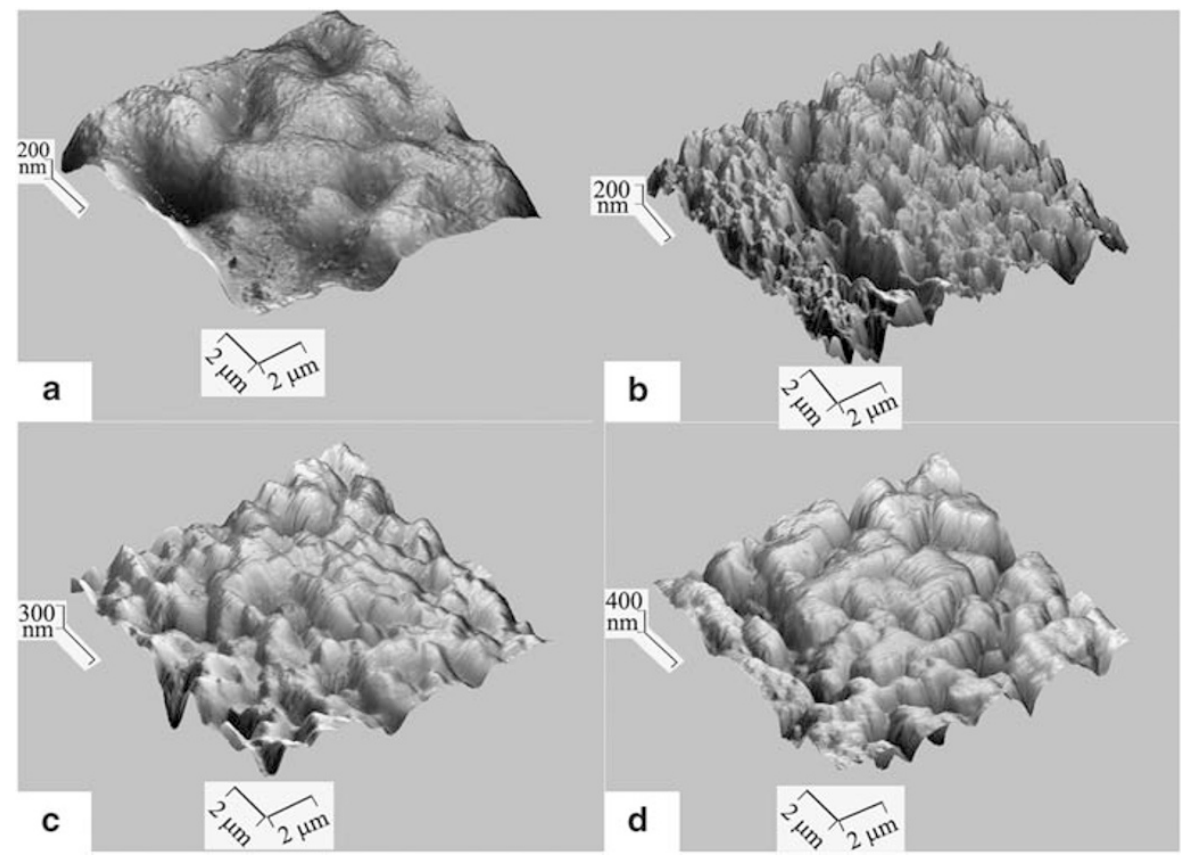

Figure 2 Atomic force microscopy topographical images of polymer films prepared from polyacrylonitrile (PAN) (a) and PAN containing 50 wt $\%$ tetrabutylammonium tetracyanoborate (b), 1-ethyl-3-methylimidazolium tetracyanoborate (c) or 1-hexyl-3-methylimidazolium tetracyanoborate (d).

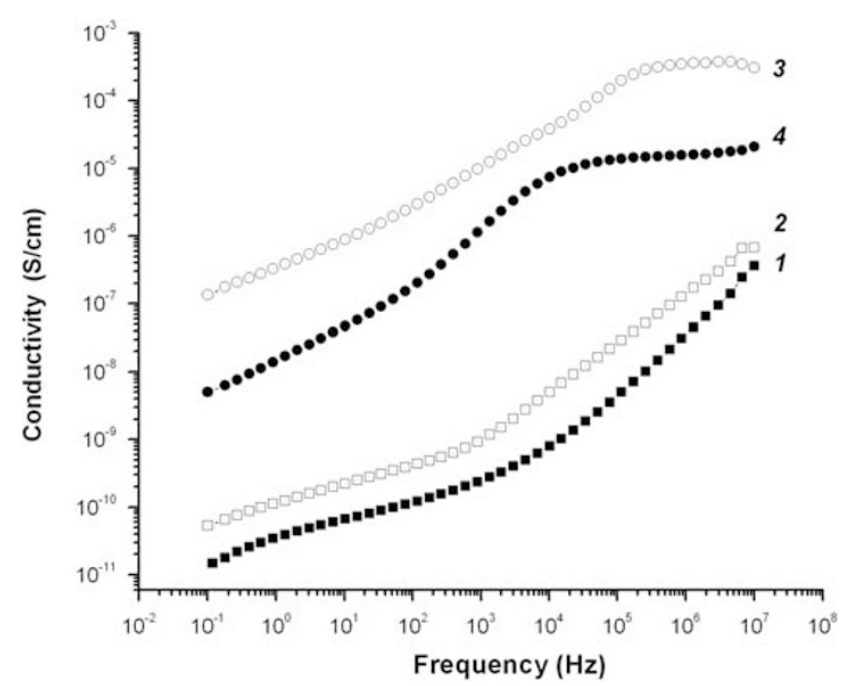

Figure 3 Frequency dependence $(\omega)$ of the ionic conductivity $(\sigma)$ at $25^{\circ} \mathrm{C}$ for the standard polyacrylonitrile (PAN) film without any filler (1) and for the films prepared from PAN containing $50 \mathrm{wt} \%$ tetrabutylammonium tetracyanoborate (2), 1-ethyl-3-methylimidazolium tetracyanoborate (3) or 1-hexyl-3-methylimidazolium tetracyanoborate (4).

by conductivity dispersion. This part of spectrum is characterized by the hopping mechanism of the charge carriers. The critical point between the plateau and the subsequent conductivity extension gives the value of the attempt rate $\omega_{\mathrm{e}}$ for the charge carriers to overcome the highest energy level. This parameter is usually assumed to give an estimate of the hopping rate of the ions and is known to be related to the $\mathrm{dc}$ conductivity $\left(\omega_{\mathrm{e}} \sim \sigma_{\mathrm{dc}}\right)$. Thus, the higher is the value of the $\mathrm{dc}$ conductivity, the higher are the frequencies where the plateau is observed. In addition to that behavior, a conductivity decrease is often observed at low frequencies, which is attributed to the electrode

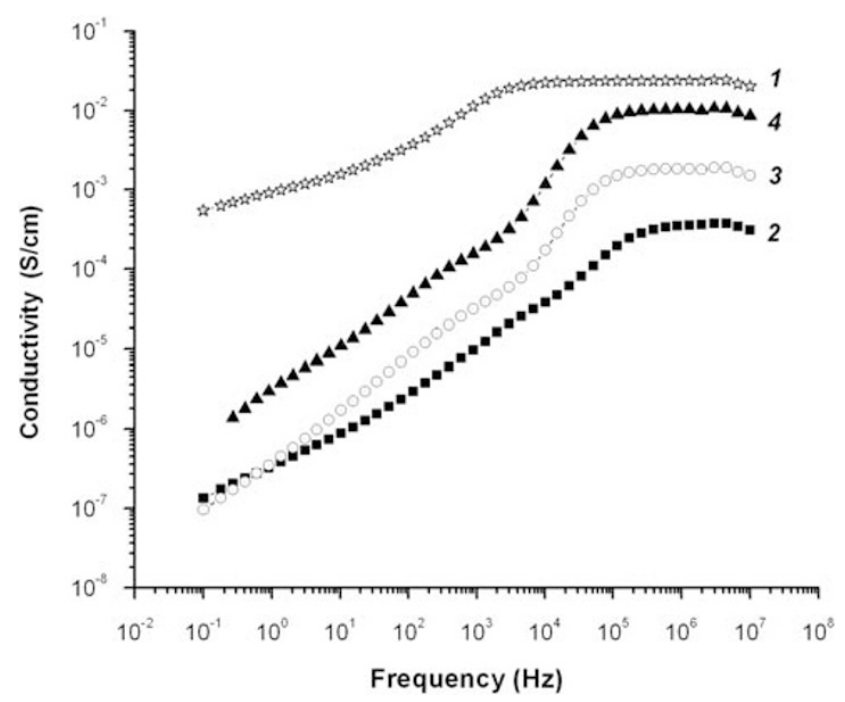

Figure 4 Frequency dependence $(\omega)$ of the ionic conductivity $(\sigma)$ at $25^{\circ} \mathrm{C}$ for pure 1-ethyl-3-methylimidazolium tetracyanoborate ionic liquid (1) and for the films prepared from polyacrylonitrile and 50 (2), 60 (3) or 70 (4) wt\% 1-ethyl-3-methylimidazolium tetracyanoborate.

polarization effects. The molecular origin of such effects is the full or partial blocking of charge carriers at the sample-electrode interface. This leads to a separation of the positive and negative charges, which gives rise to an additional polarization. The characteristic time constant of this process is proportional to the sample thickness and inversely proportional to the $\mathrm{dc}$ conductivity. Thus, the effect is shifting to the higher frequency area with the increase of the dc conductivity. In non-ideal systems at frequencies higher than the plateau region, the increase in the conductivity reflects the polarization processes, which are clearly shown in Figure 3, curves 1 
and 2. As shown in Figure 3 (curves 3 and 4) and Figure 4 (curves 1-4), the curves $\sigma^{\prime}(\omega)$ turn down to low conductivity values at frequencies below the plateau, which can be explained by the accumulation of ions on the electrode surface, that is, by the so-called electrode effects.

Comparative analysis showed that composite films filled with imidazolium tetracyanoborates possessed better conductivity than films with tetraalkyammonium tetracyanoborate (Figure 3, curves 2, 3 and 4). The $\sigma^{\prime}(\omega)$ dependence for the composite film with tetraalkylammonium tetracyanoborate was very similar to that of pure PAN, which is known to be a good dielectric material (Figure 3, curves 2 and 1, respectively). This phenomenon can be explained by the possible complexing between the PAN and the IL described above, which leads to the decreased dissociation of the latter. Thus, the ion mobility is limited and as a result the conductivity increases insignificantly in comparison with pure PAN.

The ionic conductivity of the film containing $50 \mathrm{wt} \%$ of 1-ethyl-3methylimidazolium tetracyanoborate was higher than that of the film filled with $50 \mathrm{wt} \%$ of 1-hexyl-3-methylimidazolium tetracyanoborate $\left(1.1 \times 10^{-5}\right.$ versus $3.7 \times 10^{-4} \mathrm{~S} \mathrm{~cm}^{-1}$ at $25^{\circ} \mathrm{C}$; see Table 3$)$. Obviously, this increase depends on the difference in the mobility of the cations investigated and, as a consequence, on the difference in the ionic conductivity of the respective ILs.

To increase the ionic conductivity of the composite materials, films containing 60 and $70 \mathrm{wt} \%$ of $\left[1-\mathrm{Me}-3\right.$-Etim] $\mathrm{B}(\mathrm{CN})_{4}$ were also prepared (Figure 4). Figure 4 (curves 2-4) shows that increase in IL content led to an increase in the ionic conductivity of the composite polymer material. Thus, $\sigma_{\mathrm{dc}}$ increased from $3.7 \times 10^{-4} \mathrm{~S} \mathrm{~cm}^{-1}$ at $25^{\circ} \mathrm{C}$ for the film with $50 \mathrm{wt} \%$ of IL to $1.1 \times 10^{-2} \mathrm{~S} \mathrm{~cm}^{-1}$ for the composite material containing $70 \mathrm{wt} \%$ of 1-ethyl-3-methylimidazolium tetracyanoborate. Further increase in the amount of IL added gave sticky films with a complete loss of mechanical stability.

To determine the influence of ILs on the mechanical properties of composite materials, tensile strength testing was carried out on films containing ILs and on a film prepared from pure PAN (see Table 3). In the case of films filled with ILs, a reduction in the tensile strength and in the elastic modulus was observed in comparison to the pure PAN film. The increase in the amount of IL added led to the subsequent decrease in the solidity of the composite films; that is, to the softening and lubrication of the films. Such a reduction of the mechanical stability can be explained by the plasticization of the polymer matrix with the IL. ${ }^{71-73}$

The 'direct' casting of the films was performed because of a particular interest in industrial applications. However, additional experiments were then performed. The same films with different amounts of tetracyanoborate ILs were cast using reprecipitated high-molecular weight PAN. The test results were found to be very similar to those obtained via 'direct' casting in Table 3. The possible explanation can be found in Table 1 (see for example, entries 1, 4 and 10). The isolated polymer yields in all ionic solvents containing $\mathrm{B}(\mathrm{CN})_{4}$ were in the range of $79-87 \%$, which means a high level of the monomer conversion. The invariability of the results supports the statement that, during the 'direct' casting, there were essentially no low-molecular weight oligomers, which could affect either the mechanical properties or the conductivity of the prepared films. The absence of the monomer residue was guaranteed by thorough drying of the films under a high vacuum.

\section{CONCLUSIONS}

Various ILs with four anions, namely, trifluoromethanesulfonate, trifluoroacetate, tris(pentafluoroethyl)trifluorophosphate and tetra- cyanoborate, were suggested for the substitution of the hydrolytically unstable hexafluorophosphate and tetrafluoroborate salts in the free radical polymerization of unsaturated monomers. The influence of the nature of the ILs on the radical polymerization of MMA and AN was studied. High-molecular weight PMMA and PAN were prepared in IL media. Generally, trifluoromethanesulfonate and trifluoroacetate ILs provided the best conditions for the synthesis of PMMA and PAN, respectively. Nevertheless, FAP-based ionic solvents promoted the formation of high-molecular weight PMMA, even when the polymerization proceeded under atmospheric pressure and the presence of oxygen. The PMMA polydispersity indexes were found to be in the range of 1.6-3.5, depending on the phase state (homogeneous or heterogeneous) of the polymerization process. No direct dependence between the viscosity of the IL used and the obtained polymer molecular weight was observed. It was demonstrated that the compatibility of the polymer with an IL is important for the formation of composite films with high ionic conductivity and good mechanical properties. The composite films prepared from PAN and tetracyanoborate ILs possessed $\sigma_{\mathrm{dc}}$ up to $1.0 \times 10^{-2} \mathrm{Scm}^{-1}$ at $25^{\circ} \mathrm{C}$ and are of interest for potential applications as polyelectrolytes for different types of batteries.

\section{ACKNOWLEDGEMENTS}

We thank Dr MA Batenkin (GA Rasuvaev Institute of Organometallic Chemistry Russian Academy of Sciences (IOMC RAS)) for the atomic force microscopy analysis of the surface of the films and Mrs VV Kazantseva (Laboratory of Polymeric Materials, INEOS RAS) for the measurement of the mechanical properties of the composite films.

1 Kubisa, P. Ionic liquids as solvents for polymerization processes-progress and challenges. Prog. Polym. Sci. 34, 1333-1347 (2009).

2 Xie, M., Han, H., Ding, L. \& Shi, J. Promotion of atom transfer radical polymerization and ring-opening metathesis polymerization in ionic liquids. J. Macromol. Sci. Part C Polym. Rev. 49, 315-338 (2009).

3 Strehmel, V. Selection of ionic liquids for free radical polymerization processes. Macromol. Symp. 254, 25-33 (2007).

4 Schmidt-Naake, G., Woecht, I. \& Schmalfuß, A. Ionic liquids-new solvents in the free radical polymerization. Macromol. Symp. 259, 226-235 (2007).

5 Schmidt-Naake, G., Woecht, I., Schmalfuß, A. \& Gluck, T. V. Polymer reaction engineering. Free radical polymerization in ionic liquids-solvent influence of new dimension. Macromol. Symp. 275-276, 204-218 (2009).

6 Strehmel, V., Reynaud, E., Wetzel, H., Gornitz, E. \& Laschewsky, A. Aspects of free radical polymerization of styrene and n-butylmethacrylate in imidazolium tosylates. Macromol. Symp. 275-276, 242-249 (2009).

7 Ueki, T. \& Watanabe, M. Macromolecules in ionic liquids: progress, challenges and opportunities. Macromolecules 41, 3739-3749 (2008).

8 Lu, J., Yan, F. \& Texter, J. Advanced applications of ionic liquids in polymer science. Prog. Polym. Sci. 34, 431-448 (2009)

9 Wasserscheid, P. \& Welton, T. (eds) Ionic Liquids in Synthesis, 2nd edn (Wiley-VCH, Weinheim, 2007).

10 Galinski, M., Lewandowski, A. \& Stepniak, I. Ionic liquids as electrolytes. Electrochim. Acta 51, 5567-5580 (2006)

11 Sato, T., Masuda, G. \& Takagi, K. Electrochemical properties of novel ionic liquids for electric double layer capacitor applications. Electrochim. Acta 49, 3603-3611 (2004).

12 Ohno, H. (ed.) Electrochemical Aspects of lonic Liquids (John Wiley: New York, 2005).

13 Vygodskii, Ya.S., Lozinskaya, E. I. \& Shaplov, A. S. Ionic liquids as novel promising reaction media for organic and polymer syntheses. Polym. Sci. J. Ser. C 43, 236-251 (2001).

14 Vygodskii, Ya.S., Mel'nik, O. A., Lozinskaya, E. I., Shaplov, A. S., Malyshkina, I. A., Gavrilova, N. D., Lyssenko, K. A., Antipin, M.Yu., Golovanov, D. G., Korlyukov, A. A., Ignat'ev, N. \& Welz-Biermann, U. The influence of ionic liquid's nature on free radical polymerization of vinyl monomers and ionic conductivity of the obtained polymeric materials. Polym. Adv. Technol. 18, 50-63 (2007).

15 Strehmel, V., Laschewsky, A., Wetzel, H. \& Gornitz, E. Free radical polymerization of n-butyl methacrylate in ionic liquids. Macromolecules 39, 923-930 (2006).

16 Vygodskii, Ya.S., Shaplov, A. S., Lozinskaya, E. I., Vlasov, P. S., Malyshkina, I. A., Gavrilova, N. D., Kumar, S. \& Buchmeiser, M. R. Cyclopolymerization of $N$, $\mathrm{N}$-dipropargylamines and $\mathrm{N}, \mathrm{N}$-dipropargyl Ammonium salts. Macromolecules 41, 1919-1927 (2008). 
17 Zhang, H., Hong, K. \& Mays, J. W. Synthesis of block copolymers of styrene and methyl methacrylate by conventional free radical polymerization in room temperature ionic liquids. Macromolecules 35, 5738-5741 (2002).

18 Harrison, S., Mackenzie, S. R. \& Haddleton, D. M. Pulsed laser polymerization in an ionic liquid: strong solvent effects on propagation and termination of methyl methacrylate. Macromolecules 36, 5072-5075 (2003).

19 Woecht, I., Schmidt-Naake, G., Beuermann, S., Buback, M. \& Garcia, N. Propagation kinetics of free radical polymerizations in ionic liquids. J. Polym. Sci. Part A Polym. Chem. 46, 1460-1469 (2008).

20 Woecht, I. \& Schmidt-Naake, G. Free radical copolymerization of methylmethacrylate and $\mathrm{N}$-phenyl maleimide in 1-butyl-3-methylimidazolium tetrafluoroborate. Macromol. Symp. 275-276, 219-229 (2009).

21 Andrzejewska, E., Podgorska-Golubska, M., Stepniak, I. \& Andrzejewski, M. Photoinitiated polymerization in ionic liquids: kinetics and viscosity effects. Polymer 50, 2040-2047 (2009).

$22 \mathrm{Wu}$, G. Z., Liu, Y. D. \& Long, D. W. Effects of ionic liquid $\left[\mathrm{Me}_{3} \mathrm{NC}_{2} \mathrm{H}_{4} \mathrm{OH}\right]^{+}\left[\mathrm{ZnCl}_{3}\right]^{-}$on $\gamma$-radiation polymerization of methyl methacrylate in ethanol and $\mathrm{N}, \mathrm{N}$-dimethylformamide. Macromol. Rapid. Commun. 26, 57-61 (2005).

23 Barth, J., Buback, M., Schmidt-Naake, G. \& Woecht, I. Termination kinetics of freeradical polymerization in ionic liquids. Polymer 50, 5708-5712 (2009).

24 Woecht, I. \& Schmidt-Naake, G. Influence of ionic liquids on the free radical copolymerization of styrene with acrylonitrile. e-Polymers 100, 1-17 (2007).

25 Hong, K., Zhang, J. W., Mays, A. E., Viesser, C. S., Brazel, J. D., Holbrey, J. D., Reichert, W. M. \& Rogers, R. D. Conventional free radical polymerization in room temperature ionic liquids a green approach to commodity polymers with practical advantages. Chem. Commun. 13, 1368-1369 (2002)

26 Chesnokov, S. A., Zakharina, M. Y., Shaplov, A. S., Chechet, Yu.V., Lozinskaya, E. I., Melnik, O. A., Vygodskii, Ya.S. \& Abakumov, G. A. Ionic liquids as catalytic additives for the acceleration of the photopolymerization of poly(ethylene glycol dimethacrylate)s. Polym. Int. 57, 538-545 (2008).

27 Chesnokov, S. A., Zakharina, M. Y. Shaplov, A. S., Lozinskaya, E. I., Malyshkina, I. A Abakumov, G. A., Vidal, F. \& Vygodskii, Ya.S. Photopolymerization of polyethylene glycol dimethacrylates: the influence of ionic liquids on the formulation and the properties of the resultant polymer materials. J. Polym. Sci. Part A Polym. Chem. 48, 2388-2409 (2010).

28 Johnston-Hall, G., Harjani, J. R., Scammells, P. J. \& Monteiro, M. J. RAFT-mediated polymerization of styrene in readily biodegradable ionic liquids. Macromolecules $\mathbf{4 2}$, 1604-1609 (2009).

29 Puttick, S., Irvine, D. J., Licence, P. \& Thurecht, K. J. RAFT-functional ionic liquids: towards understanding controlled free radical polymerisation in ionic liquids. J. Mater. Chem. 19, 2679-2682 (2009).

30 Perrier, S., Davis, T. P., Carmichael, A. J. \& Haddleton, D. M. Reversible addition-fragmentation chain transfer polymerization of methacrylate, acrylate and styrene monomers in 1-alkyl-3-methylimidazolium hexfluorophosphate. Eur. Polym. J. 39, 417-422 (2003).

31 Perrier, S., Davis, T. P., Carmichael, A. J. \& Haddleton, D. M. First report of reversible addition-fragmentation chain transfer (RAFT) polymerisation in room temperature ionic liquids. Chem. Commun. 19, 2226-2227 (2002).

32 Carmichael, A. C., Haddleton, D. M., Bon, S. A. F. \& Seddon, K. R. Copper(I) mediated living radical polymerisation in an ionic liquid. Chem. Commun. 14, 1237-1238 (2000).

$33 \mathrm{Ma}$, H. Y., Wan, X. H., Chen, X. F. \& Zhou, Q. F. Reverse atom transfer radical polymerization of methyl methacrylate in imidazolium ionic liquids. Polymer 44, 5311-5316 (2003)

$34 \mathrm{Ma}$, H. Y., Wan, X. H., Chen, X. F. \& Zhou, Q. F. Reverse atom transfer radical polymerization of methyl methacrylate in room-temperature ionic liquids. J. Polym. Sci. Part A Polym. Chem. 41, 143-151 (2003).

35 Li, N. J., Lu, J. M., Xu, Q. F., Xia, X. W. \& Wang, L. H. Reverse atom transfer radical polymerization of MMA via immobilized catalysts in imidazolium ionic liquids. J. Appl. Polym. Sci. 103, 3915-3919 (2007).

36 Ding, S., Radosz, M. \& Shen, Y. Ionic liquid catalyst for biphasic atom transfer radical polymerization of methyl methacrylate. Macromolecules 38, 5921-5928 (2005).

37 Chen, H., Liang, Y., Wang, M., Lv, P. \& Xuan, Y. Reverse ATRP of ethyl acrylate with ionic liquids as reaction medium. Chem. Eng. J. 147, 297-301 (2009).

38 Sarbu, T. \& Matyjaszewski, K. ATRP of methylmethacrylate in the presence of ionic liquids with ferrous and cuprous anions. Macromol. Chem. Phys. 202, 3379-3391 (2001).

39 Biedron, T. \& Kubisa, P. Atom transfer radical polymerization of acrylates in an ionic liquid: synthesis of block copolymers. J. Polym. Sci. Part A Polym. Chem. 40, 2799-2809 (2002).

40 Zhao, Y. L., Zhang, J. M., Jiang, J., Chen, C. F. \& Xi, F. Atom transfer radical copolymerization of $n$-hexylmaleimide and styrene in an ionic liquid. J. Polym. Sci. Part A Polym. Chem. 40, 3360-3366 (2002).

41 Ding, S. J., Tang, H. D., Radosz, M. \& Shen, Y. Q. Atom transfer radical polymerization of ionic liquid 2-(1-butylimidazolium-3-yl)ethyl methacrylate tetrafluoroborate. J. Polym. Sci. Part A Polym. Chem. 42, 5794-5801 (2004).

42 Biedron, T. \& Kubisa, P. Radical polymerization in a chiral ionic liquid: atom transfer radical polymerization of acrylates. J. Polym. Sci. Part A Polym. Chem. 43, 3454-3459 (2005)

43 Tang, H. D., Tang, J. B., Ding, S. J., Radosz, M. \& Shen, Y. Q. Atom transfer radical polymerization of styrenic ionic liquid monomers and carbon dioxide absorption of the polymerized ionic liquids. J. Polym. Sci. Part A Polym. Chem. 43, 1432-1443 (2005).

44 Maria, S., Biedron, T., Poli, R. \& Kubisa, P. Atom transfer radical polymerization of methyl acrylate with molybdenum halides as catalysts in an ionic liquid.. J. Appl. Polym. Sci. 105, 278-281 (2007).
45 Vijayaraghavan, R. \& MacFarlane, D. R. Group transfer polymerization in hydrophobic ionic liquids. Chem. Commun. 9, 1149-1151 (2005).

46 Biedron, T. \& Kubisa, P. Atom-transfer radical polymerization of acrylates in an ionic liquid. Macromol. Rapid Commun. 22, 1237-1242 (2001).

47 Vijayaraghavan, R. \& MacFarlane, D. R. Charge transfer polymerization in ionic liquids. Aust. J. Chem. 57, 129-133 (2004).

48 Ryan, J., Aldabbagh, F., Zetterlund, P. B. \& Yamada, B. First nitroxide-mediated controlled/living free radical polymerization in an ionic liquid. Macromol. Rapid Commun. 25, 930-934 (2004).

49 Perrier, S., Davies, P. T., Carmichael, A. J. \& Haddleton, D. M. Reversible additionfragmentation chain transfer polymerization of methacrylate, acrylate and styrene monomers in 1-alkyl-3-methylimidazolium hexfluorophosphate. Eur. Polym. J. 39, 417-422 (2003).

50 Percec, V. \& Grigoras, C. Catalytic effect of ionic liquids in the $\mathrm{Cu}_{2} \mathrm{O} / 2,2$ '-bipyridine catalyzed living radical polymerization of methyl methacrylate initiated with arenesulfonyl chlorides. J. Polym. Sci. Part A Polym. Chem. 43, 5609-5619 (2005).

51 Thurecht, K. J., Gooden, P. N., Goel, S., Tuck, C., Licence, P. \& Irvine, D. J. Free-radical polymerization in ionic liquids: the case for a protected radical. Macromolecules 41, 2814-2820 (2008)

52 Swatloski, R. P., Holbrey, J. D. \& Rogers, R. D. Ionic liquids are not always green: hydrolysis of 1-butyl-3-methylimidazolium hexafluorophosphate. Green Chem. 5, 361-363 (2003).

53 Ignat'ev, N. V., Welz-Biermann, U., Kucheryna, A., Bissky, G. \& Willner, H. New ionic liquids with tris(perfluoroalkyl)trifluorophosphate (FAP) anions. J. Fluorine Chem. 126, 1150-1159 (2005).

54 Kuang, D., Wang, P., Ito, S., Zakeeruddin, S. M. \& Grätzel, M. Stable mesoscopic dyesensitized solar cells based on tetracyanoborate ionic liquid electrolyte. J. Am. Chem. Soc. 128, 7732-7733 (2006).

55 Ignat'ev, N. V., Welz-Biermann, U. \& Willner, H. New perspective ionic liquids. J. Mendeleev Chem. Soc. (Rossiiskii Khimicheskii Zhurnal Rossiiskoe Khimicheskoe Obshchestvo im. D.I. Mendeleeva) 48, 36-39 (2004) (Chem Abstr 144:292104).

56 Vygodskii, Ya.S., Mel'nik, O. A., Lozinskaya, E. I. \& Shaplov, A. S. Free-radical polymerization of methyl methacrylate in ionic liquids. Vysokomol. Soed. (Polym. Sci. J.) Series A 46, 347-351 (2004) (Chem Abstr 2004;141:124011).

57 Susan, Md.-H., Kaneko, T., Noda, A. \& Watanabe, M. Ion gels prepared by in situ radical polymerization of vinyl monomers in an ionic liquid and their characterization as polymer electrolytes. J. Am. Chem. Soc. 127, 4976-4983 (2005).

58 Ohno, H., Yoshizawa, M. \& Ogihara, W. A new type of polymer gel electrolyte: zwitterionic liquid/polar polymer mixture. Electrochim. Acta 48, 2079-2083 (2003).

59 Tamada, M., Watanabe, T., Horiec, K. \& Ohno, H. Control of ionic conductivity of ionic liquid/photoresponsive poly(amide acid) gels by photoirradiation. Chem. Commun. 39, 4050-4052 (2007).

60 Zhou, D. Z., Spinks, G. M., Wallace, G. G., Tiyapiboonchaiya, C., MacFarlane, D. R., Forsyth, M. \& Sun, J. Solid state actuators based on polypyrrole and polymer-in-ionic liquid electrolytes. Electrochim. Acta 48, 2355-2359 (2003).

61 Ohno, H. Molten salt type polymer electrolytes. Electrochim. Acta 46, 1407-1411 (2001).

62 Washiro, S., Yoshizawa, M., Nakajima, H. \& Ohno, H. Highly ion conductive flexible films composed of network polymers based on polymerizable ionic liquids. Polymer 45, 1577-1582 (2004)

63 Ding, S., Tang, H., Radosz, M. \& Shen, Y. Atom transfer radical polymerization of ionic liquid 2-(1-butylimidazolium-3-yl)ethyl methacrylate tetrafluoroborate. J. Polym. Sci. Polym. Chem. 42, 5794-5801 (2004).

64 Harrison, S., MacKenzie, S. R. \& Haddleton, D. R. Unprecedented solvent-induced acceleration of free-radical propagation of methyl methacrylate in ionic liquids. Chem. Commun. 23, 2850-2851 (2002).

65 Rafikov, S. R., Pavlova, S. A. \& Tverdokhlebova, I. I. Methods for the Measurement of Molecular Weight and Molecular Weight Distribution of High Polymers (Acad. of Sci. USSR, Moscow, 1963) [in Russian].

66 Zhang, S., Lu, X., Zhou, Q., Li, X., Zhang, X. \& Li, S. Ionic Liquids: Physicochemical Properties (Elsevier Science Ltd, Amsterdam-Oxford, 2009).

67 Vygodskii, Ya.S., Mel'nik, O. A., Lozinskaya, E. I. \& Shaplov, A. S. Radical (co)polymerization of acrylonitrile in ionic liquids. Vysokomol. Soed. (Pol. Sci. J.), Ser. B 47, 122-126 (2005)(Chem Abstr 2005, 143, 173431).

68 Odian, G. Principles of Polymerization 4th ed. (John Wiley \& Sons, Inc., Hoboken, New Jersey, 2004).

69 Sakaebe, H. \& Matsumoto, H. N-methyl- $N$-propylpiperidinium bis(trifluoromethanesulfonyl)imide (PP13-TFSI) — novel electrolyte base for Li battery. Electrochem. Commun. 5, 594-598 (2003).

70 Kawauchi, T., Kumaki, J., Okoshi, K. \& Yasshima, E. Stereocomplex formation of isotactic and syndiotactic poly(methyl methacrylate)s in ionic liquids leading to thermoreversible ion gels. Macromolecules 38, 9155-9160 (2005).

71 Scott, M. P., Brazel, C. S., Benton, M. G., Mays, J. W., Holbrey, J. D. \& Rogers, R. D. Application of ionic liquids as plasticizers for poly(methyl methacrylate). Chem. Commun. 13, 1370-1371 (2002).

72 Scott, M. P., Rahman, M. \& Brazel, C. S. Application of ionic liquids as low-volatility plasticizers for PMMA. Eur. Polym. J. 39, 1947-1953 (2003).

73 Shaplov, A. S., Goujon, L., Vidal, F., Lozinskaya, E. I., Meyer, F., Malyshkina, I. A., Chevrot, C., Teyssie, D., Odinets, I. L. \& Vygodskii, Ya.S. Ionic IPNs as novel candidates for highly conductive solid polymer electrolytes. J. Polym. Sci. Part A Polym. Chem. 47, 4245-4266 (2009). 